\title{
Nucleation of wetting layers
}

\author{
Edgar M. Blokhuis* \\ Department of Chemistry, Baker Laboratory, Cornell University, Ithaca, New York 14853-1301
}

(Received 13 October 1994)

\begin{abstract}
Using Nakanishi and Fisher's model [Phys. Rev. Lett. 49, 1565 (1982)] for the wetting of a liquid on a substrate, we calculate the structure and free energy of critical circular domains. The first creation of critical domains denotes the onset of the nucleation of the wetting layer from a metastable surface state. It is shown that at the metastable limit, where the metastable surface state ceases to exist, the free energy and height of the critical domain vanish while the radius of the critical domain diverges. First-order corrections in an expansion in $S$, the surface tension difference between the metastable and the equilibrium surface state, to the usual thermodynamic analysis are calculated. As a result we have that, at coexistence, the nucleation time is given by $\Delta t \propto S^{8 \pi R} \exp \left(\frac{\pi \tau^{2}}{k T S}\right)$, where $\tau$ is the line tension at the wetting transition and $R$ is the rescaled surface tension of the liquid-vapor interface that is universal $(R \approx 0.10)$ near the critical temperature.
\end{abstract}

PACS number(s): 68.45.Gd, 64.60.Qb, 64.60.My, 82.60.Nh

\section{INTRODUCTION}

When two fluid phases coexist on a substrate, they can do so in two possible ways. Either the two fluid phases and the substrate meet in a line or one of the fluid phases intrudes between the substrate and the other fluid phase. In the latter case we say that one of the fluids "wets" the substrate. The transition between these two states is termed the wetting transition $[1,2]$. We will refer to the fluid phase that wets the substrate as the liquid phase while the other fluid phase will be referred to as the vapor phase. When the liquid is not stable as a bulk phase, the (first-order) wetting transition is announced as a prewetting transition at which the liquid layer on the substrate jumps from thin to thick. Above the (pre)wetting transition the thick liquid layer is the thermodynamically stable surface state, but often the thin film is encountered in experiments as a (long lived) metastable surface state [3-5]. Conversely, below the (pre)wetting transition the thin film is the equilibrium surface state and the thick film can be encountered as a metastable surface state. Recently there has been a lot of experimental [3-5] and theoretical [6-11] interest in the way the equilibrium surface state is formed from the metastable surface state. The quantity that one primarily has access to in experiments is the nucleation time, the time it takes for the metastable surface state to disappear. The first quantitative study of the nucleation time has been carried out by Law [3] for a near critical binary mixture of acetone and hexadecane in coexistence with the common vapor. The growth of the acetone rich phase, which wets

\footnotetext{
*Present address: Department of Physical and Macromolecular Chemistry, Gorlaeus Laboratories, P.O. Box 9502, 2300 RA Leiden, The Netherlands.
}

the interface between the hexadecane rich phase and the common vapor, was monitored after initially quenching the temperature from above the critical temperature $T_{c}$ to a specific temperature below $T_{c}$. Initially the nucleation time was found to always decrease approaching $T_{c}$, but a later study [8] seemed to indicate that very close to $T_{c}$ the nucleation time goes through a minimum and possibly diverges at the critical temperature.

According to classical nucleation theory [12], the equilibrium surface state is formed by the creation of circular domains of equilibrium surface state by thermal fluctuations. If the circular domain is larger than some critical domain it will grow and spread across the substrate, whereas it will decrease in size and ultimately vanish when it is smaller than the critical domain. The free energy necessary to create the critical domain is directly related to the experimentally measurable nucleation time and it is the calculation of the form and free energy of the critical domain that is the subject of the present investigation.

A thermodynamic treatment, which should be valid for large circular domains, shows that the size of the critical domain is determined by $S$, the difference in surface tension of the metastable and equilibrium surface phase, and $\tau$, the line tension associated with the circumference of the circular domain. The line tension tends to decrease the radius of the domain whereas $S$ is the force behind increasing the radius of the domain. The radius of the critical domain $R_{c}$ is determined by the balance of these two effects and is given by $S=\tau / R_{c}$, the twodimensional analog of the Laplace equation. Of course a thermodynamic treatment will not yield numerical values for $S$ and $\tau$, which can be supplied only by a more microscopic treatment. Previous calculations [6,9-11] of this sort have been carried out using the interface displacement model $[6,13,14]$. In this model the free energy is considered as a functional of the height profile $\ell(r)$, where $r$ is the radial distance from the center of the domain. Different forms for the potential that describes 
the interaction, which can be either short or long ranged, with the substrate are assumed. Extensive investigations by Bausch and Blossey [9-11] have yielded, as a function of dimensionality and as a function of the exponent $\sigma$ that describes the decay $\left(\propto \ell^{1-\sigma}\right)$ of the interaction with the substrate, the dependence on $S$ of the structure and free energy of (large) critical domains.

In the present investigation we will consider a form for the free energy, first given by Nakanishi and Fisher [15], that is a functional of the full density profile $m(r, z)$, where $z$ is the height above the substrate. The interaction with the substrate is considered to be short ranged and is given in terms of two parameters: $h_{1}$, the surface field, and $g$, the surface enhancement parameter $[15,16]$. Since we consider the free energy as a functional of the full density profile instead of a more coarse-grained height profile, this approach seems appropriate to investigate the structure and free energy of small critical domains.

In the next section we describe the mean-field model for the surface free energy by Nakanishi and Fisher. In Sec. III we use this model to calculate, numerically, the structure and free energy of the critical domain. The calculations are done close to the prewetting or the wetting transition where the domains are large, as well as far from the (pre)wetting transition where the domains are small and ultimately disappear. In Sec. IV we then investigate whether we can understand our numerical results for small domains by a more analytical calculation. We summarize and discuss our results in Sec. V.

\section{WETTING AND PREWETTING}

In this section we describe the mean-field model for the surface free energy that was first introduced by Nakanishi and Fisher [15]. This model has been extensively used for the description of the wetting behavior of a liquid and its vapor on a substrate. As a function of two phenomenological parameters $h_{1}$ and $g$, which describe the interaction of the liquid-vapor system with the substrate, a rich phase diagram is found containing both first- and second-order wetting transitions.

The surface free energy per unit area as a functional of the density $m(z)$ is, in the model by Nakanishi and Fisher, given by the expression

$$
F[m] / A=\int_{0}^{\infty} d z\left[\frac{1}{2}\left(\frac{\partial}{\partial z} m(z)\right)^{2}+f(m)\right]+\Phi\left(m_{1}\right)
$$

with the functions $f(m)$ and $\Phi\left(m_{1}\right)$ given by

$$
\begin{aligned}
f(m) & =\frac{1}{2}\left(1-m^{2}\right)^{2}-\frac{1}{2}\left(1-m_{v}^{2}\right)^{2}+h\left(m-m_{v}\right), \\
\Phi\left(m_{1}\right) & =-h_{1} m_{1}-\frac{g}{2} m_{1}^{2} .
\end{aligned}
$$

Here we have located the substrate at the $z=0$ plane and defined $m_{1} \equiv m(z=0)$ as the density at the substrate.

All the quantities in this expression are dimensionless: lengths are scaled by a factor of $2 \xi$, with $\xi$ the bulk correlation length; densities are scaled by $m_{0}$, which is defined as plus (minus) the density of the liquid (vapor) at coexistence; and energies are scaled by $2 \xi c m_{0}^{2}$, with $c / 2$ the usual coefficient of the squared gradient term. The bulk field $h(\geq 0)$ measures the distance from coexistence of the wetting phase, which we will refer to as the liquid phase, with the vapor phase. When $h=0$ the liquid and vapor phase coexist with densities $m=1$ and $m=-1$, respectively, while for $h>0$ only the vapor phase is stable as a bulk phase. The density of the vapor phase $m_{v}$ is the value of the density for which $f(m)$ is minimal and is for small $h$ given by

$$
m_{v}=-1-\frac{1}{4} h+\frac{3}{32} h^{2}-\frac{1}{16} h^{3}+O\left(h^{4}\right) .
$$

The phenomenological parameters $h_{1}$ and $g$ appearing in the expression for the surface free energy $\Phi\left(m_{1}\right)$ are the surface field and surface enhancement parameter, respectively $[15,16]$.

The equilibrium density profile is found by solving the Euler-Lagrange equations associated with the minimization of the surface free energy in Eq. (1) with the appropriate boundary condition at the substrate:

$$
\begin{aligned}
\frac{\partial^{2}}{\partial z^{2}} m(z) & =f^{\prime}(m), \\
\left.\frac{\partial}{\partial z} m(z)\right|_{z=0} & =-\left[2 f\left(m_{1}\right)\right]^{\frac{1}{2}}=\Phi^{\prime}\left(m_{1}\right),
\end{aligned}
$$

where the prime denotes a differentiation with respect to the argument. The solution to Eq. (4) can be found analytically $[17,18]$

$$
m(z)=m_{v}+\frac{m_{v}\left(3 m_{v}^{2}-1\right)}{\left(1-2 m_{v}^{2}\right) e^{z^{*}}-m_{v}^{2}+\frac{h m_{v}}{2 \alpha} \sinh \left(z^{*}\right)},
$$

where we have defined

$$
\begin{aligned}
\alpha & \equiv\left(6 m_{v}^{2}-2\right)^{\frac{1}{2}}\left(3 m_{v}^{2}-2\right)^{\frac{1}{2}}+4 m_{v}^{2}-2 \\
z^{*} & \equiv\left(6 m_{v}^{2}-2\right)^{\frac{1}{2}}(z-\ell) .
\end{aligned}
$$

The above expression for the density profile was first given by Jin and Fisher [17] in an expansion in small $h$. The height $\ell$, defined so that $m(z=\ell)=0$, is a measure of the thickness of the liquid layer on the substrate. It is explicitly given by

$$
\begin{aligned}
\ell= & \frac{-1}{\left(6 m_{v}^{2}-2\right)^{\frac{1}{2}}} \ln \left(\frac{m_{v}}{\alpha\left(m_{v}-m_{1}\right)}\right. \\
& \times\left[\left(6 m_{v}^{2}-2\right)^{\frac{1}{2}}\left(m_{1}^{2}+2 m_{v} m_{1}+3 m_{v}^{2}-2\right)^{\frac{1}{2}}\right. \\
& \left.\left.+2 m_{v} m_{1}+4 m_{v}^{2}-2\right]\right)
\end{aligned}
$$

The density at the substrate $m_{1}$ is found by solving the boundary condition in Eq. (5). A graphical representation of the boundary condition is shown in Fig. 1. The curve $\left[2 f\left(m_{1}\right)\right]^{\frac{1}{2}}$ intersects the solid line $h_{1}+g m_{1}$ at four values of $m_{1}$ of which two correspond to (local) minima of the surface free energy. These two values are indicated by the solid circles in Fig. 1 and will be denoted 


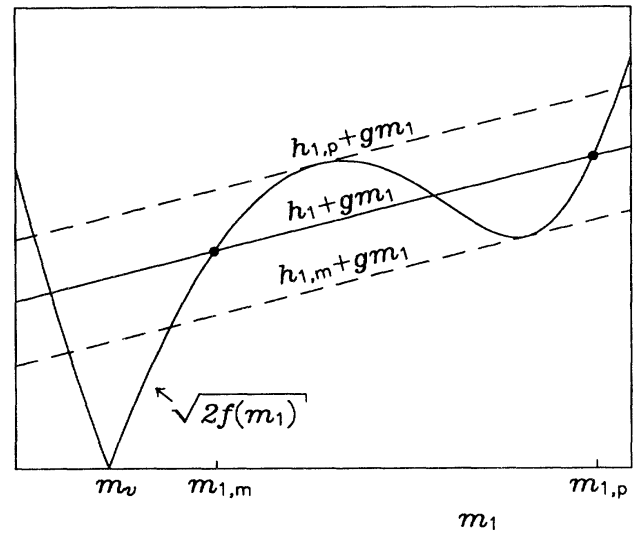

FIG. 1. Graphical representation of the boundary condition in Eq. (5). Solutions of the boundary condition are found by the intersections of the solid curve with the straight solid line. The solid circles with $m_{1}=m_{1, m / p}$ are solutions of the boundary condition that correspond to (local) minima in the surface free energy. The broken lines tangent to the solid curve represent the location of the metastable limits.

by $m_{1, m}$ and $m_{1, p}$ so that $m_{1, m}<m_{1, p}$. The surface tensions corresponding to these two minima are calculated by inserting the explicit expression for the density profile into Eq. (1)

$$
\begin{aligned}
\sigma= & \frac{1}{3}\left(m_{1}^{2}-m_{v} m_{1}-2\right)\left(m_{1}^{2}+2 m_{v} m_{1}+3 m_{v}^{2}-2\right)^{\frac{1}{2}} \\
& +h \ln \left(\frac{\left(m_{1}^{2}+2 m_{v} m_{1}+3 m_{v}^{2}-2\right)^{\frac{1}{2}}+m_{1}+m_{v}}{\left(6 m_{v}^{2}-2\right)^{\frac{1}{2}}+2 m_{v}}\right) \\
& +\frac{2}{3}\left(6 m_{v}^{2}-2\right)^{\frac{1}{2}}+\Phi\left(m_{1}\right) .
\end{aligned}
$$

The lower of the two calculated surface tensions is the equilibrium surface tension while the other surface tension corresponds to a metastable surface state. When $h=0$, i.e., when the liquid and the vapor are in coexistence, and for a fixed value of $g$, a particular $h_{1}=h_{1, W}$ exists so that for $h_{1}<h_{1, W}$ the minimum corresponding to $m_{1, m}$ has the lower surface tension while for $h_{1}>h_{1, W}$ the minimum corresponding to $m_{1, p}$ has the lower surface tension. In the latter case the corresponding thickness of the liquid layer is infinite and the transition is therefore termed the wetting transition. For $g>-2$ the wetting transition is a first-order transition, which implies that off coexistence $(h>0)$ it is announced as a prewetting transition. At the prewetting transition the equilibrium surface state changes from that of a thin liquid layer to that of a thick (but not infinitely thick as for the wetting transition) liquid layer. Along the locus of prewetting transitions, termed the prewetting line, the surface tensions of the thin and the thick liquid layer are equal and the thin and the thick layer can coexist on the substrate. The prewetting line ends in a surface critical point at which the surface states corresponding to the thin and the thick layer become identical.

From Fig. 1 it is clear that for fixed values of $g$ and $h$ only a certain range of values of $h_{1}$ yields two competing minima. The bounds on the range of values of $h_{1}$, which we will denote as $h_{1, p}$ and $h_{1, m}$ so that $h_{1, p}>h_{1, m}$, are graphically determined by the tangent lines to the curve $\left[2 f\left(m_{1}\right)\right]^{\frac{1}{2}}$ that have slope $g$ (see Fig. 1$)$. When $h_{1}>h_{1, p}$, solving the boundary condition yields $m_{1, p}$ as the only minimum in the surface free energy while for $h_{1}<h_{1, m}$ only $m_{1, m}$ corresponds to a minimum in the surface free energy. The graphical construction yields that $h_{1, p}$ and $h_{1, m}$ are the positive solutions of the following fourth-order equation in $h_{1}$ :

$$
\begin{aligned}
& h_{1}^{4}-2 m_{v} g h_{1}^{3}-\frac{1}{16}\left[g^{4}+8 g^{2}\left(5-6 m_{v}^{2}\right)+48 m_{v}^{2}-72 m_{v} h-32\right] h_{1}^{2} \\
&+\frac{g}{8}\left[m_{v} g^{4}+2 g^{2}\left(4 m_{v}-h\right)+16 m_{v}-72 m_{v}^{2} h+48 h\right] h_{1}+\frac{g^{6}}{16}\left(2-3 m_{v}^{2}\right)+\frac{g^{4}}{8}\left(6-10 m_{v}^{2}+m_{v} h\right) \\
&+\frac{g^{2}}{8}\left(12-20 m_{v}^{2}+9 h^{2}-6 m_{v} h\right)+1-m_{v}^{2}-\frac{9}{2} m_{v} h+8 m_{v}^{2} h^{2}=0 .
\end{aligned}
$$

As mentioned above, for a certain value of $h_{1}$, which we will denote as $h_{1, P W}$ (or $h_{1, W}$ when $h=0$ ), in the interval $h_{1, m}<h_{1}<h_{1, p}$, the surface tensions of the thin and the thick liquid layer are equal. The situation is thus such that for $h_{1}<h_{1, m}$ the thin film is the equilibrium state; for $h_{1, m}<h_{1}<h_{1, P W}$ the thin film is still the equilibrium state, but now the thick film enters as a metastable state; for $h_{1, P W}<h_{1}<h_{1, p}$ the thick film is the equilibrium state and now the thin film can be present as a metastable state; for $h_{1}>h_{1, p}$ the thick film is the equilibrium state. The values $h_{1, m}$ and $h_{1, p}$ denote the limits of the metastable regions.

A typical phase diagram is shown in Fig. 2 in the case that $g=0$. The solid line is the prewetting line, which starts at the wetting transition $\left(W ; h=0, h_{1}=\right.$ $\left.(2 \sqrt{3}-3)^{\frac{1}{2}} \approx 0.68125 \ldots\right)$ and ends at the surface critical point (SCP; $h=\frac{4}{9} \sqrt{3}, h_{1}=\sqrt{3}$ ). The broken lines are the limits of the metastable regions given by

$$
\begin{aligned}
h_{1, m} & =\left[\frac{9}{2} m_{v}^{4}-3 m_{v}^{2}-1+\frac{m_{v}}{2}\left(4-3 m_{v}^{2}\right)^{\frac{3}{2}}\right]^{\frac{1}{2}}, \\
h_{1, p} & =\left[\frac{9}{2} m_{v}^{4}-3 m_{v}^{2}-1-\frac{m_{v}}{2}\left(4-3 m_{v}^{2}\right)^{\frac{3}{2}}\right]^{\frac{1}{2}}
\end{aligned}
$$

which are derived from setting $g=0$ in Eq. (10). 


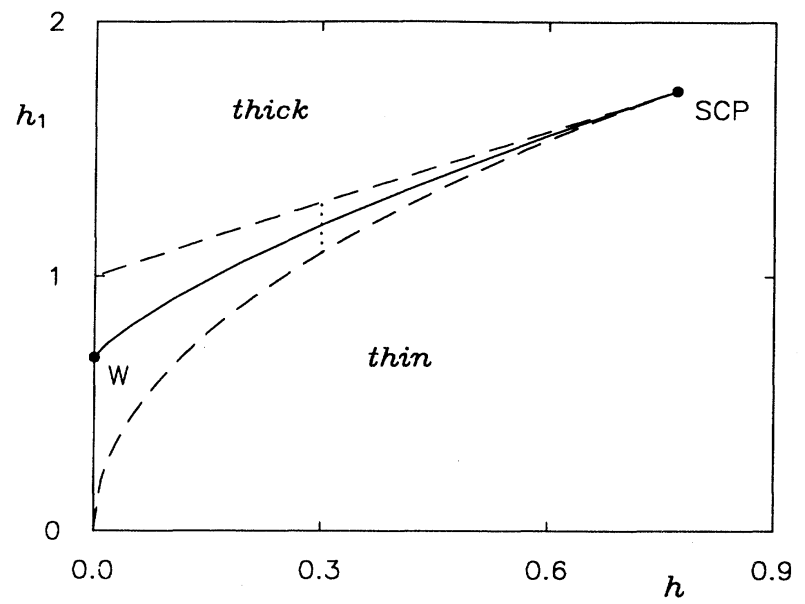

FIG. 2. Phase diagram for $g=0$. The solid curve is the prewetting line where the thickness of the wetting film jumps from thin (region below the prewetting line) to thick (region above the prewetting line). The wetting transition $(W)$ is at $h=0$ and $h_{1} \simeq 0.68125 \ldots$. The prewetting line ends in the surface critical point (SCP) at $h=\frac{4}{9} \sqrt{3}$ and $h_{1}=\sqrt{3}$. The broken curves represent the end of the metastable regions. The dotted line at $h=0.3$ represents the range of values of $h_{1}$ corresponding to Figs. 3, 4, and 6 .

In the next section we will stick to setting $g=0$ as an example. We are interested in the decay of metastable surface states to the equilibrium surface states and we will carry out our investigation in three typical regions of interest. These are the decay of a metastable thick layer and that of a thin layer for a particular $h>0(h=0.3)$ as well as the decay of a metastable thin layer when $h=0$.

\section{NUCLEATION}

With the model for the surface free energy given in the preceding section, we now want to investigate how a metastable surface state is replaced by the equilibrium surface state. In particular we will be interested in the time, the nucleation time, it takes for the equilibrium state to form. Suppose the thermodynamic circumstances are such that the thin film is the equilibrium state of the system and we change our thermodynamic variables instantly in such a way that now the thick film becomes the equilibrium state of the system. By thermal fluctuations, circular domains of the equilibrium surface state (in this example the thick film) will form that either will decrease in size and vanish or increase in size and cover the whole substrate. When these spontaneously formed domains are large enough (we will later return to what exactly we mean by "large enough"), thermodynamics tells us that the driving force behind increasing the size of the domain is $S \equiv \sigma_{\text {meta }}-\sigma_{\text {eq }}$, the difference in surface tension of the metastable and the equilibrium surface state, times the surface area of the domain, while the driving force behind decreasing the size is the line tension $\tau[14,18-22]$ times the circumference of the domain. The total free energy for the creation of a circular domain with radius $R$ is thus given by

$$
F(R)=-S \pi R^{2}+2 \pi R \tau .
$$

The radius of the critical domain is defined as the radius at which the above expression has its maximum

$$
S=\frac{\tau}{R_{c}} .
$$

This is the two-dimensional analog of Laplace's law [2]. It should be kept in mind that the above equation is derived from maximizing the free energy whereas the original Laplace equation, or its two-dimensional analog, is derived from minimizing the free energy. Whenever a domain is created with a radius larger than the critical radius it will grow to spread the whole substrate whereas it will decrease in size and vanish when its radius is smaller than the critical radius. An important quantity is the free energy needed to create the critical domain. It is calculated by inserting the critical radius into Eq. (12)

$$
F_{c}=\frac{\pi \tau^{2}}{S}
$$

The time it will take to form a critical domain by a thermal fluctuation, and thus the time it takes to form the equilibrium surface state, is inversely proportional to the probability of creating a critical domain. It is thus inversely proportional to the Boltzmann factor $\exp \left[-\beta F_{c}\right]$, where $\beta \equiv 1 / k T, k$ is Boltzmann's constant, and $T$ is the temperature. The nucleation time can be measured in experiments and thus allows us to obtain information about the behavior of $F_{c}$ and thus about $\tau$ and $S$.

In this section we will calculate the free energy of the critical domain using the model for the surface free energy in Eq. (1). This microscopic approach has the advantage that one has explicit expressions [18] for $\tau$ and $S$ and, furthermore, it has the advantage that the analysis is not restricted to large circular domains allowing us to investigate the range of validity of the thermodynamic expression in Eq. (14). Using the cylindrical symmetry of the critical domain, the density is a function of $r$, the radial distance, and $z$. The surface free energy as a functional of the density now reads

$$
F[m]=2 \pi \int_{0}^{\infty} d z \int_{0}^{\infty} d r r\left[\frac{1}{2}\left(\frac{\partial}{\partial z} m(r, z)\right)^{2}+\frac{1}{2}\left(\frac{\partial}{\partial r} m(r, z)\right)^{2}+f(m)\right]+2 \pi \int_{0}^{\infty} d r r \Phi\left(m_{1}\right)
$$


where $m_{1}(r) \equiv m(r, z=0)$. The form of the critical domain is calculated by solving the Euler-Lagrange equation for the above free energy. The Euler-Lagrange equations define an extremum in the free energy with respect to the many degrees of freedom. In the present example this extremum is a saddle point: the free energy is maximal with respect to the degree of freedom that results in a change of the radius of the domain and it is minimal with respect to all the smaller degrees of freedom [9]. This corresponds precisely to the situation described for the critical domain in Eqs. (12) and (13).

Solving the Euler-Lagrange equations amounts to solving a nonlinear second-order differential equation on a two-dimensional grid. Instead we will make an approximation that is expected to be very accurate and which will lead to solving a similar differential equation as a function of only one variable. Define the height $\ell(r)$, which now is a function of $r$, as $m(r, z=\ell(r))=0$. Instead of $r$ as a variable we can, without loss of generality, use $\ell(r)$ as a variable when there is a one to one relation between the two. The density is then written as a function of $\ell$ and $z$. With this transformation the free energy has the form

$$
F[\ell]=2 \pi \int_{0}^{\infty} d r r\left\{\frac{1}{2} \sigma_{0}(\ell)\left[\ell^{\prime}(r)\right]^{2}+V(\ell)\right\}
$$

where we have defined

$$
\begin{aligned}
\sigma_{0}(\ell) \equiv & \int_{0}^{\infty} d z\left(\frac{\partial}{\partial \ell} m(\ell, z)\right)^{2}, \\
V(\ell) \equiv & \int_{0}^{\infty} d z\left[\frac{1}{2}\left(\frac{\partial}{\partial z} m(\ell, z)\right)^{2}+f(m)\right] \\
& +\Phi\left(m_{1}\right)-\sigma_{\text {meta }} .
\end{aligned}
$$

The form of the free energy in Eq. (16) is the direct analog of the interface displacement model $[6,13,14]$ that was used by Bausch and Blossey [9-11] for their calculations of critical domains. The function $\sigma_{0}(\ell)$ denotes the surface tension against surface area fluctuations of the liquid-vapor interface located at height $\ell$. When $\ell$ is large compared to some typical interaction range of the liquid with the substrate, $\sigma_{0}(\ell)$ is expected to become equal to $\frac{4}{3}$, the surface tension of the free liquid-vapor interface. The function $V(\ell)$ is the surface potential that measures the surface free energy needed to constrain the liquid-vapor interface to be at a certain height $\ell$.

Instead of using $\ell(r)$ as parameter to replace $r$ it proves to be more convenient to use $m_{1}(r)$ as parameter. Then the density is given by $m\left(m_{1}, z\right)$ and the free energy is found by substituting $m_{1}(r)$ for $\ell(r)$ in the above formulas:

$$
F\left[m_{1}\right]=2 \pi \int_{0}^{\infty} d r r\left[\frac{1}{2} \sigma_{0}\left(m_{1}\right)\left[m_{1}^{\prime}(r)\right]^{2}+V\left(m_{1}\right)\right],
$$

where now

$$
\begin{aligned}
\sigma_{0}\left(m_{1}\right) \equiv & \int_{0}^{\infty} d z\left(\frac{\partial}{\partial m_{1}} m\left(m_{1}, z\right)\right)^{2} \\
V\left(m_{1}\right) \equiv & \int_{0}^{\infty} d z\left[\frac{1}{2}\left(\frac{\partial}{\partial z} m\left(m_{1}, z\right)\right)^{2}+f(m)\right] \\
& +\Phi\left(m_{1}\right)-\sigma_{\text {meta }} .
\end{aligned}
$$

The approximation now comes in when making certain assumptions about the form of the functions $\sigma_{0}\left(m_{1}\right)$ and $V\left(m_{1}\right)$. In order to obtain explicit expressions for these functions, we need to postulate a form for the density profile $m\left(m_{1}, z\right)$. We will use the density profile $[17,18]$

$$
m\left(m_{1}, z\right)=m_{v}+\frac{m_{v}\left(3 m_{v}^{2}-1+\lambda\right)}{\left(1-\lambda-2 m_{v}^{2}\right) e^{z_{\lambda}^{*}}-m_{v}^{2}+\frac{m_{v}}{2 \alpha_{\lambda}}\left(h-2 m_{v} \lambda\right) \sinh \left(z_{\lambda}^{*}\right)}
$$

where we have defined

$$
\begin{aligned}
\alpha_{\lambda} \equiv & \left(6 m_{v}^{2}-2+2 \lambda\right)^{\frac{1}{2}}\left(3 m_{v}^{2}-2+2 \lambda\right)^{\frac{1}{2}}+4 m_{v}^{2}-2+2 \lambda \\
z_{\lambda}^{*} \equiv & \left(6 m_{v}^{2}-2+2 \lambda\right)^{\frac{1}{2}}(z-\ell) \\
\ell= & \frac{-1}{\left(6 m_{v}^{2}-2+2 \lambda\right)^{\frac{1}{2}}} \ln \left(\frac{m_{v}}{\alpha_{\lambda}\left(m_{v}-m_{1}\right)}\right. \\
& \times\left[( 6 m _ { v } ^ { 2 } - 2 + 2 \lambda ) ^ { \frac { 1 } { 2 } } \left(m_{1}^{2}+2 m_{v} m_{1}+3 m_{v}^{2}-2\right.\right. \\
& \left.\left.+2 \lambda)^{\frac{1}{2}}+2 m_{v} m_{1}+4 m_{v}^{2}-2+2 \lambda\right]\right)
\end{aligned}
$$

and $\lambda$ in terms of $m_{1}$ is found by solving the modified boundary condition

$$
-\left[2 f\left(m_{1}\right)+2 \lambda\left(m_{1}-m_{v}\right)^{2}\right]^{\frac{1}{2}}=\Phi^{\prime}\left(m_{1}\right) .
$$

Several different expressions for $m\left(m_{1}, z\right)$ and hence the functions $\sigma_{0}\left(m_{1}\right)$ and $V\left(m_{1}\right)$ have been presented in the literature and we refer the reader to Refs. [17,22,23] for their form. As an aside we mention that the above profile is obtained from minimizing the surface free energy in Eq. (1) constraining the density at the substrate to be equal to a prescribed value $m_{1}$ by adding a term containing a Lagrange multiplier of the form [17]

$$
\lambda \int_{0}^{\infty} d z\left[m(z)-m_{v}\right]^{2}
$$

An important advantage of this approach is that the expression for the density profile in Eq. (20) is compact. The functions $V\left(m_{1}\right)$ and $\sigma_{0}\left(m_{1}\right)$ can now be calculated by inserting the density profile in Eq. (20) into Eq. (19). The integral over $z$ in the expression for the surface potential $V\left(m_{1}\right)$ can be carried out to yield 


$$
\begin{aligned}
V\left(m_{1}\right)= & \frac{1}{3}(2+\lambda)\left(6 m_{v}^{2}-2+2 \lambda\right)^{\frac{1}{2}}+\left(m_{1}^{2}+2 m_{v} m_{1}+3 m_{v}^{2}-2+2 \lambda\right)^{\frac{1}{2}} \\
& +h \ln \left(\frac{\left(m_{1}^{2}+2 m_{v} m_{1}+3 m_{v}^{2}-2+2 \lambda\right)^{\frac{1}{2}}+m_{1}+m_{v}}{\left(6 m_{v}^{2}-2+2 \lambda\right)^{\frac{1}{2}}+2 m_{v}}\right)+\frac{1}{3}\left(m_{1}^{2}-m_{v} m_{1}-2-\lambda\right)+\Phi\left(m_{1}\right)-\sigma_{\text {meta }}
\end{aligned}
$$

The Euler-Lagrange equation corresponding to the free energy in Eq. (18) reads

$$
\begin{aligned}
V^{\prime}\left(m_{1}\right)= & \sigma_{0}\left(m_{1}\right) m_{1}^{\prime \prime}(r)+\frac{1}{2} \sigma_{0}^{\prime}\left(m_{1}\right)\left[m_{1}^{\prime}(r)\right]^{2} \\
& +\frac{1}{r} \sigma_{0}\left(m_{1}\right) m_{1}^{\prime}(r) .
\end{aligned}
$$

The profile $m_{1}(r)$ of the critical domain is obtained from the above differential equation with the boundary conditions $m_{1}^{\prime}(r=0)=0$ and $m_{1}(r=\infty)=m_{1, \text { meta }}$, where $m_{1, \text { meta }}$ is the density at the substrate of the metastable surface state. This then enables us to calculate $\ell(r)$ the height profile of the critical domain using Eqs. (21) and (22). In Fig. 3 a number of height profiles of critical domains are shown for $g=0, h=0.3$, and, from top to bottom, $h_{1}=1.21,1.22,1.23, \ldots, 1.29$. The prewetting transition is at $h_{1, P W}=1.201712 \ldots$ so that for this range of values of $h_{1}$ the thick film is the equilibrium surface state and the thin film the metastable surface state. The critical domains thus consist of thick film in an environment of thin film surface state. In Fig. 4 a number of height profiles of critical domains are shown for $g=0, h=0.3$, and, from top to bottom, $h_{1}=1.10$, $1.11,1.12, \ldots, 1.19$. For this range of values of $h_{1}$ the thin film is the equilibrium surface state and the thick film the metastable surface state. The critical domains are now critical "dents" consisting of thin film in an environment of thick film surface state. The critical domains

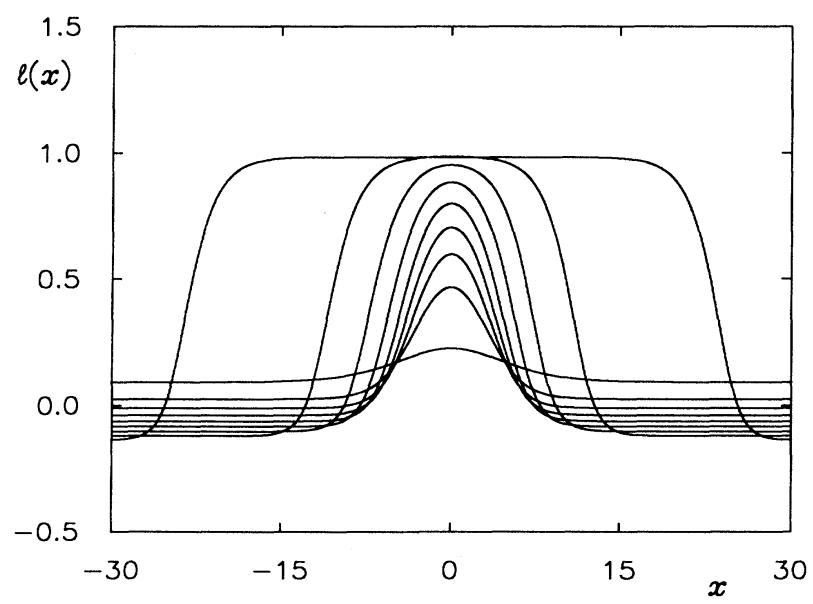

FIG. 3. Cross section of the critical domain for $g=0$, $h=0.3$, and, from top to bottom, $h_{1}=1.21,1.22,1.23,1.24$, $1.25,1.26,1.27,1.28$, and 1.29. Shown is the height profile $\ell(x)$ with $x$ parallel to the substrate. in Figs. 3 and 4 are larger when $h_{1}$ is close to the the prewetting transition while the critical domains decrease in size close to the metastable limits, which are located at $h_{1, m}=1.093855 \ldots$ and $h_{1, p}=1.290951 \ldots$. Notice that only the largest domains exhibit a clear plateau of the equilibrium surface state inside the domain. We will later see that only for these largest domains is the thermodynamic analysis, leading to the expression for the free energy of the critical domain in Eq. (14), valid.

In Fig. 5 a number of height profiles of critical domains are shown for $g=0, h=0$ (liquid-vapor coexistence), and, from top to bottom, $h_{1}=0.71,0.72,0.73,0.75,0.77$, $0.80,0.85,0.90,0.95$, and 0.98 . The wetting transition is at $h_{1, W}=0.68125 \ldots$ so that for this range of values of $h_{1}$ the thick film, which is now infinitely thick, is the equilibrium surface state and the thin film the metastable surface state. The metastable limit is located at $h_{1, p}=$ 1. Since the equilibrium thickness is infinitely thick, the height $\ell(r)$ never reaches a plateau [11] inside the domain and in Sec. IV we will investigate the consequences of this fact for the thermodynamic analysis.

The free energy of the critical domains is calculated by inserting the height profiles from Figs. 3-5 into the expression for the free energy in Eq. (16). The result, as a function of $h_{1}$, is shown as the solid circles for $h=0.3$ in Fig. 6 and for $h=0$ in Fig. 7. The free energy of the critical domain on either side of the (pre)wetting transition diverges near the (pre)wetting transition. This can be understood from the fact that at the (pre)wetting

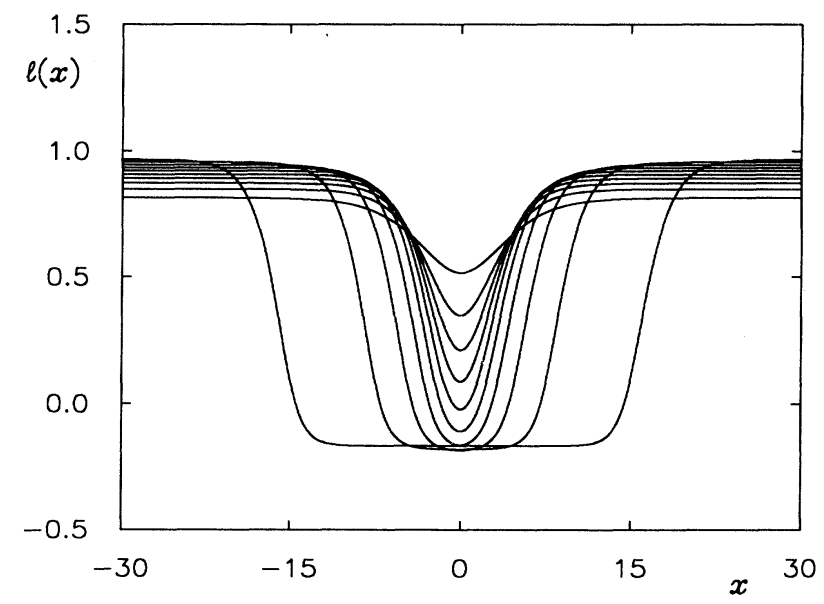

FIG. 4. Cross section of the critical domain for $g=0$, $h=0.3$, and, from top to bottom, $h_{1}=1.10,1.11,1.12,1.13$, $1.14,1.15,1.16,1.17,1.18$, and 1.19 . Shown is the height profile $\ell(x)$ with $x$ parallel to the substrate. 


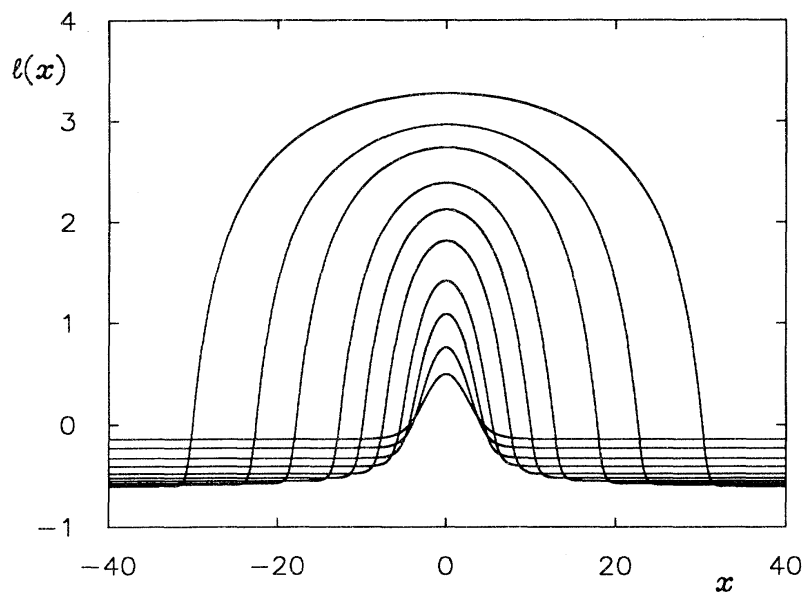

FIG. 5. Cross section of the critical domain for $g=0, h=0$, and, from top to bottom, $h_{1}=0.71,0.72,0.73,0.75,0.77$, $0.80,0.85,0.90,0.95$, and 0.98 . Shown is the height profile $\ell(x)$ with $x$ parallel to the substrate.

transition the difference $S$ in surface tensions between the equilibrium and the metastable surface state goes to zero as $S=\Delta m_{1,(P) W}\left|h_{1}-h_{1,(P) W}\right|$, where $\Delta m_{1,(P) W}$ is the difference in densities at the substrate between the thick and thin film at the (pre)wetting transition. Near the (pre)wetting transition we thus have from Eq. (14) that

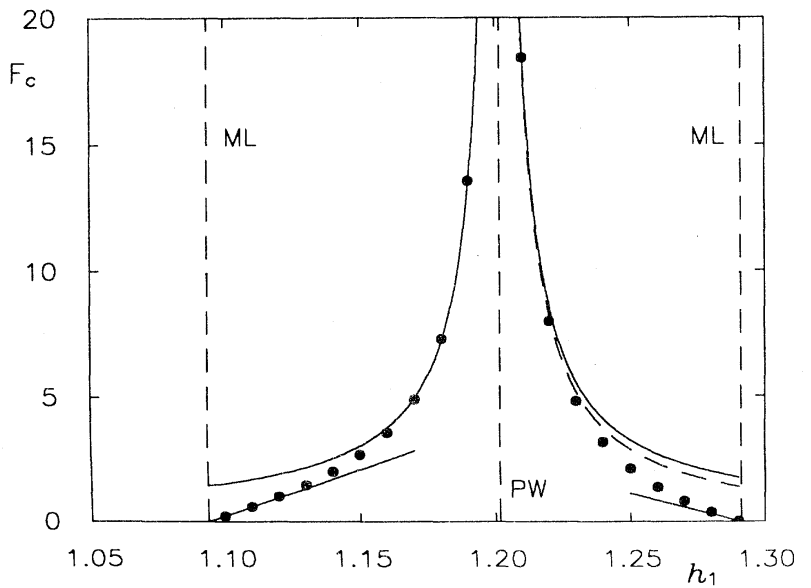

FIG. 6. Free energy of the critical domains as a function of $h_{1}$ for $g=0$ and $h=0.3$ (dotted line in Fig. 2). The solid circles are the free energies calculated from the height profiles in Figs. 3 and 4 . The solid curves are the free energies calculated from the thermodynamic formula in Eq. (26), which describes the leading-order behavior near the prewetting transition (PW). The broken curve is the first-order correction to the thermodynamic formula (see Sec. IV B). The straight solid lines are the leading-order behavior near the metastable limits (ML).

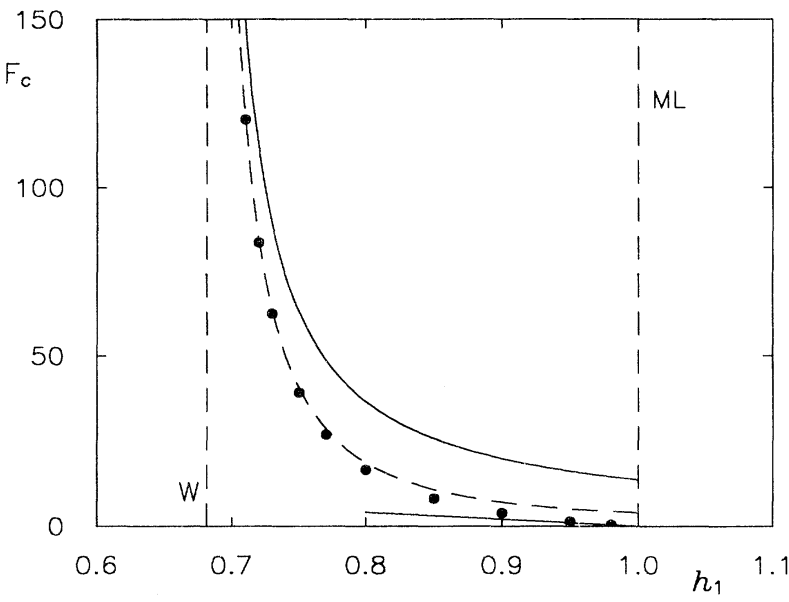

FIG. 7. Free energy of the critical domains as a function of $h_{1}$ for $g=0$ and $h=0$. The solid circles are the free energies calculated from the height profiles in Fig. 5. The solid curve is the free energy calculated from the thermodynamic formula in Eq. (26), which describes the leading-order behavior near the wetting transition $(W)$. The broken curve is the leading-order correction to the thermodynamic formula [see Eq. (51)]. The straight solid line is the leading-order behavior near the metastable limit (ML).

$$
F_{c}=\frac{\pi \tau^{2}}{\Delta m_{1,(P) W}} \frac{1}{\left|h_{1}-h_{1,(P) W}\right|}+\cdots
$$

The solid curves in Fig. 6 are the free energy of the critical domain calculated using the above formula with $\tau=0.259103 \ldots$ and $\Delta m_{1, P W}=1.347397 \ldots$. It is observed that a good fit is obtained for the large domains, but that for the smaller domains, especially near the metastable limits, significant differences are apparent. The solid curve in Fig. 7 is the free energy of the critical domain calculated using the above formula with $\tau=\tau_{W}=1.602 \ldots$ and $\Delta m_{1, W}=1.8612097 \ldots$. The relative difference between the solid circles and the solid curve only goes to zero for very large domains while the absolute value of the difference even diverges. Notice also the increase in scale of the free energy between Figs. 6 and 7 . It can be largely attributed to the dramatic increase in the value of the line tension as $h \rightarrow 0(\tau=1.602$ compared to $\tau=0.259103$ ).

It is clear from the results in Figs. 6 and 7 that the thermodynamic formula for $F_{c}$ in Eq. (14) has only a limited range of validity. In the next section we want to investigate improvements on the thermodynamic formula. First we will investigate the behavior of the free energy of the critical domain near the metastable limit. The numerical results seem to indicate that $F_{c}=0$ at the metastable limit. Second, we want to investigate the form of the leading-order correction to the thermodynamic formula for the free energy of the critical domain in an expansion around the (pre)wetting transition. 


\section{SMALL CRITICAL DOMAINS}

In this section we will investigate what happens near the metastable limits and calculate the leading-order correction to the thermodynamic formula for the free energy of the critical domain given in Eq. (14). For the description of nucleation of three-dimensional droplets [12], leading-order corrections to the thermodynamic expression for the free energy of the critical droplet define a quantity termed the Tolman length [24]. We will therefore refer to the leading order corrections to the formula in Eq. (14) as Tolman corrections.

\section{A. Metastable limit}

Close to the metastable limit the height profile $\ell(r)$ is close to the value of the height of the metastable surface phase for all $r$; see Figs. 3-5. The same is true for the profile $m_{1}(r)$, which we expand around the density at the substrate of the metastable surface phase

$$
m_{1}(r)=m_{1, m}+\Delta m_{1}(r)
$$

Here, for simplicity, we have taken the thin film to be the metastable surface state, corresponding to the situation in Figs. 3 and 5. The analysis for the case when the thick film is the metastable surface state (Fig. 4) is analogous to that of the thin film. The constant value of the density at the substrate of the thin film is itself expanded around its value at the metastable limit

$$
m_{1, m}=m_{1, \mathrm{ML}}-\Delta m_{1, m} .
$$

Next we expand the surface potential $V\left(m_{1}\right)$ around its (local) minimum value $V\left(m_{1, m}\right)=0$

$$
\begin{aligned}
V\left(m_{1}\right)= & \frac{1}{2} V^{\prime \prime}\left(m_{1, m}\right) \Delta m_{1}^{2}+\frac{1}{6} V^{\prime \prime \prime}\left(m_{1, m}\right) \Delta m_{1}^{3}+\cdots \\
= & -\frac{1}{2} V^{\prime \prime \prime}\left(m_{1, \mathrm{ML}}\right) \Delta m_{1, m} \Delta m_{1}^{2} \\
& +\frac{1}{6} V^{\prime \prime \prime}\left(m_{1, \mathrm{ML}}\right) \Delta m_{1}^{3}+\cdots
\end{aligned}
$$

where we have used the fact that at the metastable limit the local minimum of the surface potential ceases to exist and becomes a saddle point, i.e., $V^{\prime \prime}\left(m_{1, M L}\right)=0$. We now insert Eqs. (27)-(29) into the Euler-Lagrange equations in Eq. (25) and expand to lowest order in $\Delta m_{1, m}$

$$
\begin{aligned}
\sigma_{0}\left(m_{1, M L}\right) \Delta m_{1}^{\prime \prime}(r)+\frac{1}{2} \sigma_{0}^{\prime}\left(m_{1, M L}\right)\left[\Delta m_{1}^{\prime}(r)\right]^{2}+\frac{1}{r} \sigma_{0}\left(m_{1, M L}\right) \Delta m_{1}^{\prime}(r) & =-V^{\prime \prime \prime}\left(m_{1, M L}\right) \Delta m_{1, m} \Delta m_{1}+\frac{1}{2} V^{\prime \prime \prime}\left(m_{1, M L}\right) \Delta m_{1}^{2}
\end{aligned}
$$

The proper scaling behavior of $\Delta m_{1}(r)$ and $r$ for small $\Delta m_{1, m}$ is given by

$$
\begin{aligned}
\Delta m_{1}(r) & =\Delta m_{1, m} f(x), \\
r & =\left(\Delta m_{1, m}\right)^{-\frac{1}{2}} x,
\end{aligned}
$$

where $f(x)$ and $x$ are the rescaled substrate density and the distance, respectively. Inserting the above expressions into the Euler-Lagrange equation in Eq. (30) and retaining only the leading-order correction for small $\Delta m_{1, m}$, one finds

$$
f^{\prime \prime}(x)=-\frac{1}{x} f^{\prime}(x)+\frac{V^{\prime \prime \prime}\left(m_{1, M L}\right)}{\sigma_{0}\left(m_{1, M L}\right)} f(x)\left[\frac{1}{2} f(x)-1\right] .
$$

The above differential equation has to be solved numerically with the boundary conditions $f^{\prime}(x=0)=0$ and $f(x=\infty)=0$. The free energy of the critical domain is then calculated from inserting the numerically obtained profile $f(x)$ into Eq. (18) using Eqs. (27)-(29) and (31)

$$
\begin{aligned}
F_{c}= & \left(\Delta m_{1, m}\right)^{2} \pi \int_{0}^{\infty} d x x\left\{\sigma_{0}\left(m_{1, M L}\right)\left[f^{\prime}(x)\right]^{2}\right. \\
& \left.-V^{\prime \prime \prime}\left(m_{1, M L}\right)[f(x)]^{2}+\frac{1}{3} V^{\prime \prime \prime}\left(m_{1, M L}\right)[f(x)]^{3}\right\} .
\end{aligned}
$$

From the above expression we see that the free energy of the critical domain goes to zero at the metastable limit proportionally to $\left(\Delta m_{1, m}\right)^{2}$. Notice from Eq. (31) that while the height of the critical domain above the metastable thin film goes to zero near the metastable limit $\left[\ell(0) \propto \Delta m_{1}(0) \propto \Delta m_{1, m}\right]$, the radius of the domain diverges $\left[r \propto\left(\Delta m_{1, m}\right)^{-\frac{1}{2}}\right]$. In fact, while the free energy of the critical domain becomes zero, the volume, and thus the number of particles constituting the critical domain, is constant $\left[V \propto \ell(0) r^{2} \propto 1\right]$. A similar phenomenon is observed [12] for three-dimensional droplets near the metastable limit. In this case the density difference between the inside and the outside of the droplet goes to zero while the radius of the droplet diverges with the result that the number of particles constituting the critical droplet remains constant.

For fixed $g$ we have that $\Delta m_{1, m} \propto\left(h_{1, p}-h_{1}\right)^{\frac{1}{2}}$ so that the free energy of the critical domain vanishes pro- 
portionally to $h_{1, p}-h_{1}$. The straight lines near the metastable limits in Figs. 6 and 7 show the result of the above analysis. In calculating the slope of the straight solid lines, we have used the fact that for $g=0$ the relation between $\Delta m_{1, m}$ and $h_{1, p}-h_{1}$ is explicitly given by

$$
\Delta m_{1, m}=\frac{\left(2 h_{1, p}\right)^{\frac{1}{2}}\left(h_{1, p}-h_{1}\right)^{\frac{1}{2}}}{\left(4-3 m_{v}^{2}\right)^{\frac{1}{4}}\left[-3 m_{v}-\left(4-3 m_{v}^{2}\right)^{\frac{1}{2}}\right]^{\frac{1}{2}}},
$$

where $h_{1, p}$ is given in Eq. (11). From Figs. 6 and 7 it is clear that only very close to the metastable limit is the free energy of the critical domain well approximated by the straight solid lines. Especially when $h=0$ (Fig. 7) there seems to be a large region where neither solid line represents the solid circles accurately.

\section{B. Tolman corrections}

We will now investigate the first-order correction to the thermodynamic formula in Eq. (14). The thermodynamic formula is valid when the radius of the domain is large compared to the thickness of the interfacial region between the thin and thick film. For small domains the radius of the domain becomes important and the line tension will in general depend on the radius of the domain so that $\tau$ in Eq. (12) has to be replaced by $\tau(R)$. Finding the critical radius by extremizing the modified Eq. (12) then yields

$$
S=\frac{\tau\left(R_{c}\right)}{R_{c}}+\frac{\partial}{\partial R_{c}} \tau\left(R_{c}\right) .
$$

We now assume that the radius-dependent line tension for large radii can be expanded in $1 / R_{c}$

$$
\tau\left(R_{c}\right)=\tau_{0}+\tau_{1} \frac{1}{R_{c}}+O\left(\frac{1}{R_{c}^{2}}\right) .
$$

Here $\tau_{0}$ is the line tension at the (pre)wetting transition, which was previously denoted as $\tau$ but we have now added the subscript 0 to distinguish it from the radiusdependent line tension $\tau(R)$. Inserting the above expression into Eq. (35) gives

$$
S=\frac{\tau_{0}}{R_{c}}+O\left(\frac{1}{R_{c}^{3}}\right)
$$

as the two-dimensional analog of the Laplace equation. The fact that the coefficient of the $1 / R_{c}^{2}$ term in this expression vanishes when the expansion in Eq. (36) is made was already known from the study of cylindrical surfaces in three dimensions and from circular surfaces in two dimensions [25]. Inserting Eqs. (36) and (37). into Eq. (12) yields for the free energy of the critical domain

$$
F_{c}=\frac{\pi \tau_{0}^{2}}{S}+2 \pi \tau_{1}+O\left(\frac{1}{R_{c}}\right)
$$

We will now set out to calculate the (constant) first-order correction $2 \pi \tau_{1}$. The radius-dependent line tension is given by inserting the equilibrium profile $m_{1}(r)$ into Eq. (18), subtracting the surface tension contributions, and dividing by the circumference $2 \pi R_{c}$ of the boundary line

$$
\tau\left(R_{c}\right)=\int_{0}^{\infty} d r \frac{r}{R_{c}}\left[\frac{1}{2} \sigma_{0}\left(m_{1}\right) m_{1}^{\prime}(r)^{2}+\Delta V\left(m_{1}\right)\right],
$$

where we have defined $\Delta V\left(m_{1}\right) \equiv V\left(m_{1}\right)-\left(\sigma_{\text {eq }}-\right.$ $\left.\sigma_{\text {meta }}\right) \theta\left(R_{c}-r\right)$ with $\theta$ the Heaviside function. Note that $V\left(m_{1}\right)$ had been defined such that $V\left(m_{1}\right)=0$ when $r \rightarrow \infty$ and we therefore do not need to subtract a similar contribution for $r>R_{c}$. Note, furthermore, that $\Delta V\left(m_{1}\right)$ thus depends on the precise location of the radius $R_{c}$. Below we will show that the quantities $\tau_{0}$ and $\tau_{1}$, however, do not depend on the precise location of the radius. Next we expand the quantities appearing in the above formula in $1 / R_{c}$

$$
\begin{gathered}
m_{1}(r)=m_{1,0}(r)+m_{1,1}(r) \frac{1}{R_{c}}+O\left(\frac{1}{R_{c}^{2}}\right), \\
\sigma_{0}\left(m_{1}\right)=\sigma_{0,0}\left(m_{1}\right)+\sigma_{0,1}\left(m_{1}\right) \frac{1}{R_{c}}+O\left(\frac{1}{R_{c}^{2}}\right), \\
\Delta V\left(m_{1}\right)=V_{0}\left(m_{1}\right)+\Delta V_{1}\left(m_{1}\right) \frac{1}{R_{c}}+O\left(\frac{1}{R_{c}^{2}}\right) .
\end{gathered}
$$

Here we have omitted the $\Delta$ before the zeroth-order term in the expansion of $\Delta V\left(m_{1}\right)$ since $\sigma_{\text {meta }}=\sigma_{\text {eq }}$ when $R_{c} \rightarrow$ $\infty$. Inserting the expanded quantities into Eq. (39) and comparing the result to the form for the radius-dependent line tension in Eq. (36), we have that

$$
\begin{aligned}
\tau_{0}= & \int_{-\infty}^{\infty} d \zeta\left[\frac{1}{2} \sigma_{0,0}\left(m_{1,0}\right)\left(m_{1,0}^{\prime}\right)^{2}+V_{0}\left(m_{1,0}\right)\right] \\
\tau_{1}= & \int_{-\infty}^{\infty} d \zeta\left\{\zeta\left[\frac{1}{2} \sigma_{0,0}\left(m_{1,0}\right)\left(m_{1,0}^{\prime}\right)^{2}+V_{0}\left(m_{1,0}\right)\right]\right. \\
& +\frac{1}{2} \sigma_{0,1}\left(m_{1,0}\right)\left(m_{1,0}^{\prime}\right)^{2}+\Delta V_{1}\left(m_{1,0}\right)+V_{0}^{\prime}\left(m_{1,0}\right) m_{1,1} \\
& \left.+\frac{1}{2} \sigma_{0,0}^{\prime}\left(m_{1,0}\right) m_{1,1}\left(m_{1,0}^{\prime}\right)^{2}+\sigma_{0,0}\left(m_{1,0}\right) m_{1,0}^{\prime} m_{1,1}^{\prime}\right\},
\end{aligned}
$$

where we have defined $\zeta \equiv r-R_{c}$ and omitted $\zeta$ as the argument of the functions $m_{1,0}$ and $m_{1,1}$. The EulerLagrange equation in Eq. (15) is also expanded in $1 / R_{c}$. To lowest order we find that

$$
\sigma_{0,0}\left(m_{1,0}\right) m_{1,0}^{\prime \prime}+\frac{1}{2} \sigma_{0,0}^{\prime}\left(m_{1,0}\right)\left(m_{1,0}^{\prime}\right)^{2}=V_{0}^{\prime}\left(m_{1,0}\right),
$$

which is integrated to yield

$$
\frac{1}{2} \sigma_{0,0}\left(m_{1,0}\right)\left(m_{1,0}^{\prime}\right)^{2}=V_{0}\left(m_{1,0}\right) .
$$

Next we insert the above expression for $V_{0}\left(m_{1,0}\right)$ into the expressions for $\tau_{0}$ and $\tau_{1}$ and partially integrate the terms containing $m_{1,1}$ in $\tau_{1}$. This gives us

$$
\tau_{0}=\int_{-\infty}^{\infty} d \zeta\left[\sigma_{0,0}\left(m_{1,0}\right)\left(m_{1,0}^{\prime}\right)^{2}\right]
$$




$$
\begin{aligned}
\tau_{1}= & \int_{-\infty}^{\infty} d \zeta\left[\zeta \sigma_{0,0}\left(m_{1,0}\right)\left(m_{1,0}^{\prime}\right)^{2}\right. \\
& \left.+\frac{1}{2} \sigma_{0,1}\left(m_{1,0}\right)\left(m_{1,0}^{\prime}\right)^{2}+\Delta V_{1}\left(m_{1,0}\right)\right]
\end{aligned}
$$

Both expressions are independent of the choice for the location of the radius of the critical domain $(\zeta=0)$. For $\tau_{0}$ this is apparent since all the quantities in the above equation are independent of a shift in $\zeta \rightarrow \zeta+\Delta \zeta$ while for $\tau_{1}$ this can be deduced when one uses the fact that $\tau_{0}=\left(\sigma_{\text {meta }}-\sigma_{\text {eq }}\right)_{1}$, the two-dimensional analog of the Laplace equation. Notice that the expressions for $\tau_{0}$ and $\tau_{1}$ only depend on $m_{1,0}$, the density profile of the critical domain with infinite radius, which is determined from the first-order differential equation in Eq. (44). In fact, the formula for the line tension $\tau_{0}$ at the prewetting transition was already used in Sec. III when we evaluated $\tau$ in Eq. (26) for Figs. 6 and 7. The result of evaluating the above expression for $\tau_{1}$ is shown in Fig. 8 for several values of $h$ along the prewetting line. At the surface critical point $\tau_{1}=0$ and at $h=0.3$ we have $\tau_{1}=0.060783 \ldots$. We can now use this last result to calculate, for $h=0.3$, the first-order correction to the expression for the free energy in Eq. (26) using Eq. (38). The result is shown in Fig. 6 as the broken curve. The (constant) correction to the solid curve is small and only for a very limited range of values for $h_{1}$ is the broken curve a significant improvement to the solid curve.

When $h \rightarrow 0$ we see from Fig. 8 that $\tau_{1}$ increases sharply. In the Appendix we show that in fact $\tau_{1}$ diverges as

$$
\tau_{1}=\frac{2}{3} \ln (h) \quad(h \rightarrow 0)
$$

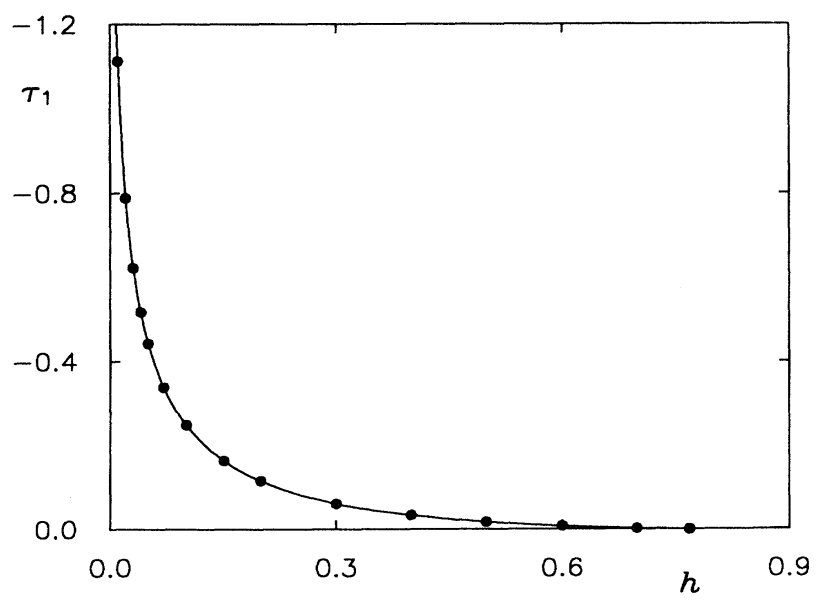

FIG. 8. Tolman correction to the line tension of the critical domain as a function of $h$ along the prewetting line (see Fig. 2 ). At the surface critical point $\tau_{1}$ becomes zero whereas near the wetting transition $\tau_{1}$ diverges as $\tau_{1}=\frac{2}{3} \ln (h)$.
The implication is that the expansion of $\tau\left(R_{c}\right)$ in $1 / R_{c}$ in Eq. (36) is no longer correct beyond the zeroth-order term, when $h=0$. In the Appendix it is shown that the correct expansion of $\tau\left(R_{c}\right)$ is given by

$$
\tau\left(R_{c}\right)=\tau_{W}-\frac{4}{3} \frac{1}{R_{c}} \ln \left(R_{c}\right)+O\left(\frac{1}{R_{c}}\right) \quad(h=0),
$$

where the coefficient of the $\ln \left(R_{c}\right) / R_{c}$ term, $-\frac{4}{3}$, is independent of the value of $g$. The two-dimensional analog of the Laplace equation is calculated by inserting the above expansion into Eq. (35)

$$
S=\frac{\tau_{W}}{R_{c}}-\frac{4}{3} \frac{1}{R_{c}^{2}}+O\left(\frac{1}{R_{c}^{3}}\right) \quad(h=0) .
$$

In this case the coefficient of the $1 / R_{c}^{2}$ term does not vanish. Inserting Eqs. (48) and (49) into Eq. (12) yields for the free energy of the critical domain

$$
F_{c}=\frac{\pi \tau_{W}^{2}}{S}+\frac{8 \pi}{3} \ln (S)+O(1) \quad(h=0) .
$$

In terms of an expansion in $h_{1}-h_{1, W}$, the zeroth- and the first-order contribution to the free energy of the critical domain are explicitly given by

$$
\begin{aligned}
F_{c}= & \frac{\pi \tau_{W}^{2}}{\Delta m_{1, W}} \frac{1}{\left|h_{1}-h_{1, W}\right|} \\
& +\frac{8 \pi}{3} \ln \left|h_{1}-h_{1, W}\right|+O(1) \quad(h=0) .
\end{aligned}
$$

The above expression for $F_{c}$ is plotted as the broken curve in Fig. 7. The addition of the first-order correction to Eq. (26) greatly improves the comparison with the calculated values represented by the solid circles.

As mentioned in Sec. II, all the quantities calculated here are in reduced units. For instance, the surface tension of the liquid-vapor interface is given by $\tilde{\sigma}=$ $\frac{4}{3} \mathrm{~cm}_{0}^{2}(2 \xi)^{-1}$, where the quantities in regular units are denoted by a tilde. The free energy of the critical domain, multiplied by $\beta$, is thus given by

$$
\beta \tilde{F}_{c}=\frac{2 \xi c m_{0}^{2}}{k T} F_{c}=\frac{3 \tilde{\sigma} \xi^{2}}{k T} F_{c}=3 R F_{c}
$$

where we have defined $\tilde{\sigma} \equiv R k T / \xi^{2}$ ( $R$ should not be confused with $R_{c}$, the radius of the critical domain). Near the critical point of the liquid-vapor system, $R$ is a $u n i$ versal constant with the experimentally determined value of $R=0.10$ [26]. The implication of Eq. (52) is that, since the coefficient of the $\ln \left(R_{c}\right) / R_{c}$ term in Eq. (48) is itself a universal constant with value $-\frac{4}{3}$, the first-order correction to the free energy of the critical domain in Eq. (50) has the universal form

$$
\beta \tilde{F}_{c, 1}=8 \pi R \ln (S) \quad(h=0) .
$$

In real systems, a description in terms of just the parameters $h_{1}$ and $g$ might not always be accurate, but we do expect that universal features, such as the expression in the above equation, will still be valid. 


\section{SUMMARY AND DISCUSSION}

We have presented numerical calculations of the structure and free energy of critical domains using the Nakanishi-Fisher [15] expression as a model free energy. For large domains our results are in agreement with the thermodynamic treatment and the findings of Bausch and Blossey [9-11] for short ranged interaction with the substrate. We have shown that at the metastable limit the free energy and the height of the critical domain vanish while the radius of the critical domain diverges. It should be kept in mind, however, that the experimentally observed metastable limit occurs prior to the theoretically calculated metastable limit (spinodal). At the experimentally observed metastable limit the kinetics of the layer growth mechanism becomes important $[12,27]$. The result is that very close to the theoretically calculated metastable limit the analysis presented here no longer holds. It would be interesting to investigate in what way our results for the structure of the critical domain (decreasing height and increasing radius with constant volume) are important for the kinetic treatment.

We have calculated first-order corrections in an expansion in $1 / R_{c}$ to the usual thermodynamic analysis. We showed that including the first-order correction to the formula for the nucleation time, at coexistence, gives

$$
\Delta t=A S^{8 \pi R} e^{\frac{\pi \tau^{2}}{k T S}}
$$

where $A$ is a constant and the above quantities are in regular units (we have omitted the tildes). The first-order correction term comes in as the factor $S^{8 \pi R}$ in the above expression. The exponent of this factor has a universal value $(\approx 2.5)$ near the critical point. It was shown (see Fig. 7) that the inclusion of this factor greatly improved the comparison with the numerically obtained values for a large range of domain sizes.

In real systems, a description in terms of just the parameters $h_{1}$ and $g$ might not always be accurate. The third phase might not be a solid substrate but a fluid phase as, for instance, when we have a binary liquid mixture with a common vapor. Also, it might be important that in real systems the interaction is usually described by a Lennard-Jones potential and the assumption of short rangedness might not always be correct. When the interaction with the substrate is sufficiently short ranged, however, we do expect universal features, such as the exponent $8 \pi R$ in the above expression, still to be valid.

Finally, we have ignored the effect of gravity. The presence of gravity will affect the structure of the large critical domains when their size becomes comparable to the capillary length. Furthermore, at coexistence, gravity will bound the thickness of the equilibrium wetting layer. For very large domains, i.e., close to the wetting transition, gravity will thus affect the analysis leading to the logarithmic correction in Eq. (53) and crossover behavior is to be expected.

\section{ACKNOWLEDGMENTS}

The author gratefully acknowledges interesting discussions with B. Widom and B. Law. D.J. Bukman, J.O. In- dekeu, and $\mathrm{B}$. Widom are thanked for a critical reading of the manuscript. The research of the author has been made possible by financial support of the Royal Netherlands Academy of Arts and Sciences. This work was carried out in the research group of B. Widom and is supported by the National Science Foundation and the Cornell University Materials Science Center.

\section{APPENDIX: CALCULATION OF $\tau_{1}$ CLOSE TO WETTING}

In this Appendix we calculate the leading behavior of $\tau_{1}$ when the wetting transition is approached $(h \rightarrow 0)$ as well as the correct expansion in $1 / R_{c}$ of the line tension $\tau\left(R_{c}\right)$ when $h=0$. First, we rewrite the expression for $\tau_{1}$ in Eq. (46), using Eq. (44) to replace integrations over $\zeta$ by integrations over $m_{1,0}$. The result is

$$
\begin{aligned}
\tau_{1}= & \int_{m_{1, m, 0}}^{m_{1, p, 0}} d m_{1,0}\left[\left(\sigma_{0,0} V_{0}\right)^{\frac{1}{2}}\left\{\int_{m_{1,0}}^{m_{1,0}^{*}} d m_{1}^{\prime}\left(\frac{\sigma_{0,0}}{V_{0}}\right)^{\frac{1}{2}}\right\}\right. \\
& \left.+\sigma_{0,1}\left(\frac{V_{0}}{2 \sigma_{0,0}}\right)^{\frac{1}{2}}+\Delta V_{1}\left(\frac{\sigma_{0,0}}{2 V_{0}}\right)^{\frac{1}{2}}\right],
\end{aligned}
$$

where we have not written the explicit dependence of the functions $\sigma_{0,0}, \sigma_{0,1}, V_{0}$, and $V_{1}$ on $m_{1,0}$. The density at the substrate of the thick film is defined by

$$
m_{1, p} \equiv m_{1, p, 0}+m_{1, p, 1} \frac{1}{R_{c}}+O\left(\frac{1}{R_{c}^{2}}\right)
$$

and similarly for $m_{1, m}$. When $h \rightarrow 0$, the limit that we will be primarily interested in, $m_{1, p, 0}$ and $m_{1, m, 0}$ are explicitly given by

$$
\begin{aligned}
& m_{1, p, 0}(h=0)=\frac{1}{2} g+\frac{1}{2}\left(g^{2}+4 h_{1}+4\right)^{\frac{1}{2}}, \\
& m_{1, m, 0}(h=0)=-\frac{1}{2} g-\frac{1}{2}\left(g^{2}-4 h_{1}+4\right)^{\frac{1}{2}} .
\end{aligned}
$$

In order to keep the analysis here as general as possible we have not set $g=0$ as we did earlier. We will show that the quantities we are interested in will be independent of $g$. Finally, in Eq. (A1) we have introduced $m_{1,0}^{*}$ as the value of $m_{1}$ where $\zeta=0\left(r=R_{c}\right)$. The value of $m_{1,0}^{*}$ is chosen arbitrarily in the range $m_{1, m, 0}<m_{1,0}^{*}<m_{1, p, 0}$.

When $h \rightarrow 0$, the singular contributions to $\tau_{1}$ are when $m_{1,0} \rightarrow m_{1, p, 0}$ so that it is convenient to use $x \equiv m_{1, p, 0}-$ $m_{1,0}$ as a variable instead of $m_{1,0}$. Equation (A1) then becomes

$$
\begin{aligned}
\tau_{1}= & \int_{0}^{\Delta m_{1,0}} d x\left[\left(\sigma_{0,0} V_{0}\right)^{\frac{1}{2}}\left\{\int_{x^{*}}^{x} d x^{\prime}\left(\frac{\sigma_{0,0}}{V_{0}}\right)^{\frac{1}{2}}\right\}\right. \\
& \left.+\sigma_{0,1}\left(\frac{V_{0}}{2 \sigma_{0,0}}\right)^{\frac{1}{2}}+\Delta V_{1}\left(\frac{\sigma_{0,0}}{2 V_{0}}\right)^{\frac{1}{2}}\right]
\end{aligned}
$$

where $\Delta m_{1,0} \equiv m_{1, p, 0}-m_{1, m, 0}$. Next we expand the functions $\sigma_{0}\left(m_{1}\right)$, as given in Eq. (19), with Eqs. (20)-(22) and $V\left(m_{1}\right)$, as given in Eq. (24), in small $x$ and $h$, with 
arbitrary ratio between the two. The result is

$$
\begin{aligned}
\sigma_{0,0}(x) & =\frac{1}{3} \frac{\hat{V}^{2}}{h^{2}} \frac{1}{\left(1+\frac{\hat{V}}{h} x\right)^{2}}, \\
V_{0}(x) & =\left[\frac{\hat{V}}{h} x-\ln \left(1+\frac{\hat{V}}{h} x\right)\right] h, \\
\sigma_{0,1}(x) & =-\frac{2}{3} m_{1, p, 1}(h=0) \frac{\hat{V}^{3}}{h^{3}} \frac{1}{\left(1+\frac{\hat{V}}{h} x\right)^{3}}, \\
\Delta V_{1}(x) & =\hat{V} m_{1, p, 1}(h=0) \frac{\frac{\hat{V}}{h} x}{\left(1+\frac{\hat{V}}{h} x\right)}
\end{aligned}
$$

where we have defined $\hat{V} \equiv 4 m_{1, p, 0}(h=0)\left[m_{1, p, 0}(h=\right.$ $0)-1] /\left[m_{1, p, 0}(h=0)+1\right]$. Next we insert the above expressions into the expression for $\tau_{1}$ in Eq. (A4) and redefine $x \rightarrow \frac{h}{\hat{V}} x$. The resulting $\tau_{1}$ is written as the sum of three terms

$$
\begin{aligned}
\tau_{1, a}= & -\frac{1}{3} \int_{1}^{1 / h} d x \frac{1}{x^{\frac{1}{2}}}\left\{\int_{x}^{\infty} d x^{\prime} \frac{1}{\left(x^{\prime}\right)^{\frac{3}{2}}}\right\}+O(1) \\
= & \frac{2}{3} \ln (h)+O(1), \\
\tau_{1, b}= & -\frac{1}{3} \sqrt{6} m_{1, p, 1}(h=0) \hat{V} \frac{1}{h^{\frac{1}{2}}} \\
& \times \int_{0}^{\infty} d x \frac{[x-\ln (x+1)]^{\frac{1}{2}}}{(x+1)^{2}}+O(1), \\
\tau_{1, c}= & \frac{1}{6} \sqrt{6} m_{1, p, 1}(h=0) \hat{V} \frac{1}{h^{\frac{1}{2}}} \\
& \times \int_{0}^{\infty} d x \frac{x[x-\ln (x+1)]^{-\frac{1}{2}}}{(x+1)^{2}}+O(1) .
\end{aligned}
$$

Only in the first expression is the (first) upper integration limit not replaced by the $h \rightarrow 0$ limit of $\infty$. This integration namely leads to the logarithmic divergence of $\tau_{1, a}$. The second and third expressions diverge as $h^{-\frac{1}{2}}$, but the coefficients can be seen to exactly cancel each other when one uses the fact that

$$
\begin{gathered}
\int_{0}^{\infty} d x \frac{2 \ln (x+1)-x}{[x-\ln (x+1)]^{\frac{1}{2}}(x+1)^{2}} \\
=\left[\frac{2[x-\ln (x+1)]^{\frac{1}{2}}}{(x+1)}\right]_{0}^{\infty}=0 .
\end{gathered}
$$

As a result, we thus find that the leading singular contribution to $\tau_{1}$ as $h \rightarrow 0$ is given by $\tau_{1, a}$

$$
\tau_{1}=\frac{2}{3} \ln (h)+O(1) .
$$

An important finding is that the coefficient of the above expression is independent of $g$ and $h_{1}$.

Next we want to investigate what is the correct expansion of the line tension in $1 / R_{c}$ when $h=0$. When $h>0$ we know that for a large enough radius of the domain the value of $m_{1}$ (or $\ell$ ) inside the domain is exponentially close to $m_{1, \text { eq }}$ (or $\ell_{\mathrm{eq}}$ ), the values of the equilibrium surface state (see Figs. 3 and 4 ). When $h=0$, however, this is not the case (see Fig. 5). The relation between the radius $R_{c}$ and the substrate density in the middle of the domain $m_{1,0}(0)$ is calculated by inserting the expressions for $\sigma_{0,0}$ and $V_{0}$ in Eq. (A5) with $h=0$ into Eq. (44) to yield

$$
\begin{aligned}
R_{c} & =\int_{m_{1,0}(0)}^{m_{1,0}^{*}} d m_{1,0}\left(\frac{\sigma_{0,0}}{2 V_{0}}\right)^{\frac{1}{2}} \\
& \approx \int_{m_{1, p, 0}-m_{1,0}(0)}^{m_{1, p, 0}-m_{1,0}^{*}} d x x^{-\frac{3}{2}} \\
& \propto\left[m_{1, p, 0}-m_{1,0}(0)\right]^{-\frac{1}{2}} .
\end{aligned}
$$

From the previous analysis we know that only $\tau_{1, a}$ contributes to the singular part of $\tau_{1}$ so that the expansion of $\tau\left(R_{c}\right)$ is given by

$$
\begin{aligned}
\tau\left(R_{c}\right)= & \tau_{W}+\frac{1}{R_{c}} \int_{m_{1, m, 0}}^{m_{1,0}(0)} d m_{1,0}\left(\sigma_{0,0} V_{0}\right)^{\frac{1}{2}} \\
& \times \int_{m_{1,0}}^{m_{1,0}^{*}} d m_{1}^{\prime}\left(\frac{\sigma_{0,0}}{V_{0}}\right)^{\frac{1}{2}} \\
= & \frac{1}{R_{c}} \int_{m_{1, p, 0}-m_{1,0}(0)}^{\Delta m_{1,0}} d x\left(\sigma_{0,0} V_{0}\right)^{\frac{1}{2}} \int_{x^{*}}^{x} d x^{\prime}\left(\frac{\sigma_{0,0}}{V_{0}}\right)^{\frac{1}{2}}
\end{aligned}
$$

Next we insert Eq. (A9) and the expressions for $\sigma_{0,0}$ and $V_{0}$ in Eq. (A5) with $h=0$ into the above expression, so that we finally arrive at

$$
\begin{aligned}
\tau\left(R_{c}\right)= & \tau_{W}-\frac{1}{3 R_{c}} \int_{1 / R_{c}^{2}}^{\Delta m_{1}} d x \frac{1}{x^{\frac{1}{2}}}\left\{\int_{x}^{x^{*}} d x^{\prime} \frac{1}{\left(x^{\prime}\right)^{\frac{3}{2}}}\right\} \\
& +O\left(\frac{1}{R_{c}}\right) \\
= & -\frac{4}{3} \frac{1}{R_{c}} \ln \left(R_{c}\right)+O\left(\frac{1}{R_{c}}\right)
\end{aligned}
$$

This is the correct expansion in $1 / R_{c}$ when $h=0$ to replace Eq. (36).
[1] M. Schick, in Liquids at Interfaces, Proceedings of the Les Houches Summer School of Theoretical Physics, Course XLVIII, edited by J. Charvolin, J.F. Joanny, and J. ZinnJustin (Elsevier, Amsterdam, 1990).

[2] J.S. Rowlinson and B. Widom, Molecular Theory of Capillarity (Clarendon, Oxford, 1982).
[3] B.M. Law, Phys. Rev. Lett. 69, 1781 (1992).

[4] J.E. Rutledge and P. Taborek, Phys. Rev. Lett. 69, 937 (1992).

[5] D. Bonn, H. Kellay, and J. Meunier (unpublished).

[6] J.F. Joanny and P.G. de Gennes, J. Colloid Interface Sci. 111, 94 (1986). 
[7] M. Schick and P. Taborek, Phys. Rev. B 46, 7312 (1992).

[8] B.M. Law, Phys. Rev. Lett. 72, 1698 (1994).

[9] R. Bausch and R. Blossey, Europhys. Lett. 15, 125 (1991).

[10] R. Bausch and R. Blossey, Z. Phys. B 86, 273 (1992).

[11] R. Bausch and R. Blossey, Phys. Rev. E 48, 1131 (1993).

[12] See, e.g., D.W. Oxtoby, in Fundamentals of Inhomogeneous Fluids, edited by D. Henderson (Dekker, New York, 1992), and references therein.

[13] N.V. Churaev, V.M. Starov, and B.V. Derjaguin, J. Colloid Interface Sci. 89, 16 (1982).

[14] J.O. Indekeu, Physica A 183, 439 (1992).

[15] H. Nakanishi and M.E. Fisher, Phys. Rev. Lett. 49, 1565 (1982).

[16] D.J. Durian and C. Franck, Phys. Rev. Lett. 59, 555 (1987).
[17] A.J. Jin and M.E. Fisher, Phys. Rev. B 47, 7365 (1993).

[18] E.M. Blokhuis, Physica A 202, 402 (1994).

[19] I. Szleifer and B. Widom, Mol. Phys. 75, 925 (1992).

[20] S. Perković, I. Szleifer, and B. Widom, Mol. Phys. 80, 729 (1993).

[21] For a review, see J.O. Indekeu, Int. J. Mod. Phys. B 8, 309 (1994).

[22] S. Perković, E.M. Blokhuis, and G. Han, J. Chem. Phys. 102, 400 (1995).

[23] D.J. Bukman, J.O. Indekeu, G. Langie, and G. Backx, Phys. Rev. B 47, 1577 (1993).

[24] R.C. Tolman, J. Chem. Phys. 17, 333 (1949).

[25] J.R. Henderson and J.S. Rowlinson, J. Phys. Chem. 88, 6484 (1984).

[26] M.R. Moldover, Phys. Rev. A 31, 1022 (1985).

[27] J. Langer, Ann. Phys. (N.Y.) 41, 108 (1967). 